\title{
A Slant Helix Characterization in Riemann-Otsuki Space
}

\author{
MÜneVver YildiRIM Yilmaz*, Mihriban KÜlahCI \\ AND AlPER O. ÖĞRENMIŞ
}

\begin{abstract}
In this paper, we establish new characterization for a slant helix from the view point of Riemann-Otsuki space.
\end{abstract}

\section{Introduction}

One of the principal goals of the study of curve theories is to develop new and alternative characterization for helices. Simply a helix in Euclidean 3space is a curve of constant slope. The study of this curve dates from 1802 with Lancret's well-known statement"A curve is a helix if and only if ratio of curvature to torsion is constant" [6]. From past to nowadays numerous scientists have studied on this magic subject from different point of view, $[1,7,2,5,9]$.

On the other hand Otsuki spaces, firstly introduced by T. Otsuki and A. Moor, have interesting properties because of supposing the relation

$$
\nabla_{k} g_{i j}=\gamma_{k} g_{i j}
$$

where $g_{i j}$ and $\gamma_{k}$ denote Riemannian metric tensor and a recurrance tensor, respectively. If $\nabla_{k} g_{i j}=0$ holds, the space called Riemann-Otsuki space. Riemann-Otsuki space is studied by various authors by different aspects $[3,4,8]$.

In [3] the author considered Riemann-Otsuki space and determined the Frenet formula with respect to the covariant and contravariant part of the connection.

The present study deals with the helices of special type in Riemann-Otsuki space. Making use of this Frenet formula we obtain new characterizations for a slant helix in Riemann-Otsuki space.

2010 Mathematics Subject Classification. 53B05, 53B15, 53B30.

Key words and phrases. Riemann-Otsuki space, Frenet Formula, slant helix.

${ }^{*}$ Corresponding author. 


\section{Preliminaries}

In Otsuki spaces, we are given a-priori given tensor $P_{j}^{i}$ such that det $\left\|P_{j}^{i}\right\| \neq 0$ holds. $Q_{j}^{i}$ is the inverse tensor that satisfies $P_{j}^{i} Q_{r}^{j}=\delta_{r}^{i}$ with respect to the local coordinates $x^{i}$ of an n-dimensional differentiable manifold,

In addition for metric Otsuki spaces the metric tensor $g_{i j}\left(\operatorname{det}\left\|g_{i j}\right\| \neq 0\right)$ is given by a relation for $\nabla_{k} g_{i j}$. In W-O ${ }_{n}$ (Weyl-Otsuki space) this relation satisfies

$$
\nabla_{k} g_{i j}=\gamma_{k} g_{i j}
$$

but in $R-O_{n}$ (Riemann-Otsuki space)

$$
\nabla_{k} g_{i j}=0
$$

holds. In Otsuki spaces the covariant differential of the tensor $T_{j}^{i}$ is defined by

$$
D T_{j}^{i}=P_{a}^{i} P_{b}^{j} \bar{D} T_{b}^{a}=P_{a}^{i} P_{b}^{j}\left(\partial_{k} T_{b}^{a}+{ }^{\prime} \Gamma_{r k}^{a} T_{b}^{r}-{ }^{\prime \prime} \Gamma_{b k}^{r} T_{r}^{a}\right) d x^{k} .
$$

The Leibnitz formula does not hold for this differential. Here we denote the basic covariant differential by $\bar{D}$. The characteristic of the Otsuki spaces are the different coefficients. We may show it as follows

$$
\left.\delta_{j}^{i}\right|_{k}={ }^{\prime} \Gamma_{j k}^{i}-{ }^{\prime \prime} \Gamma_{j k}^{i} \neq 0 .
$$

The coefficient of the connection " $\Gamma_{j k}^{i}$ is determined from the relation (1.1). For the coefficients of connection ${ }^{\prime} \Gamma_{j k}^{i}$ we use the following equation which is known as Otsuki's relation;

$$
\partial_{k} P_{j}^{i}+{ }^{\prime \prime} \Gamma_{a k}^{i} P_{j}^{a}-P_{a}^{i^{\prime}} \Gamma_{j k}^{a}=0 .
$$

In Otsuki spaces it is possible to determine the covariant differentials $D$ and $\bar{D}$ with respect only covariant and contravariant parts of the connection. So

$$
{ }^{\prime} \bar{D} T_{j}^{i}=\nabla_{k} T_{j}^{i} d x^{k}=\left(\partial_{k} T_{j}^{i}+{ }^{\prime} \Gamma_{r k}^{i} T_{j}^{r}-{ }^{\prime} \Gamma_{j k}^{r} T_{r}^{i}\right) d x^{k}
$$

holds. For this basic covariant differential the Leibnitz formula holds. The basic covariant differential " $\bar{D}$ can be defined in the same way.

It is characteristic that the basic covariant differential ' $\bar{D}$ is identical in the case of contravariant indices with the basic covariant differential $\bar{D}$, and similarly in the case of covariant indices the basic covariant differential " $\bar{D}$ is identical with the basic covariant differential $\bar{D}$. 
We give the basic relations as follows [3]

$$
\begin{aligned}
{ }^{\prime} \bar{D} g_{i j} & =d g_{i j}-\left({ }^{\prime} \Gamma_{i k}^{r} g_{r j}+{ }^{\prime} \Gamma_{j k}^{r} g_{i r}\right) d x^{k}, \\
{ }^{\prime \prime} \bar{D} g_{i j} & =d g_{i j}-\left({ }^{\prime \prime} \Gamma_{i k}^{r} g_{r j}+{ }^{\prime \prime} \Gamma_{j k}^{r} g_{i r}\right) d x^{k}=0, \\
{ }^{\prime} \bar{D} g^{r a} & =-g^{i a} g^{j r}\left({ }^{\prime} \bar{D} g_{i j}\right), \\
{ }^{\prime \prime} \bar{D} g^{r a} & =0 .
\end{aligned}
$$

In [3], the author considered the Frenet formula with respect to the contravariant and covariant components of the vectors in detail. In addition the Frenet formula for the covariant part is not different from the well-known formula of the Riemannian case. Hence we only consider the contravariant components of the vectors for Riemann-Otsuki space. For a deeper understanding we refer to [3].

\subsection{The Frenet Formula with Respect to the Contravariant Com-} ponents of the Vectors. We can take a point $M$ of the curve $C: x^{i}=$ $x^{i}(s)$ where $\mathrm{s}$ is the arclength parameter. For point $M$ the components of the unit tangent vector $v_{0}$ are given as $v_{0}^{i}=\frac{d x^{i}}{d s}$.

Theorem 1.1. If $C: x^{i}(s)$ is the curve of an $R-O_{n}$ space and $v_{l}, l=$ $0, \ldots, p-1(p<n)$ are mutually orthogonal unit vectors which satisfy the following relation

$$
\bar{D} v_{l} j=-\kappa_{l} v_{l-1}^{j}+\kappa_{l+1} v_{l+1}^{j}+v_{l}^{r} \bar{D} \delta_{r}^{j}
$$

such that

$$
\begin{cases}\kappa_{q}=0, & \text { for } q=0, \\ \kappa_{q}=\left(g_{i j}\left(\bar{D}_{q-1} v^{j}+\kappa_{q-1} v_{q-2}^{j}\right)\right), & \text { for } q=1, \ldots, p-1 .\end{cases}
$$

Here $v_{p+1}$ is the unit vector orthogonal to all before and $\kappa_{0}=0$ and $\kappa_{n}=0$ holds. Then the vector $v_{p}$ satisfies the relation, (1.10), too.

If we use Otsuki's covariant differential $D$, then from the connection $D_{v}^{j}=$ $P_{a}^{j} \bar{D} v^{a}$ it follows that $\bar{D} v^{a}=Q_{i}^{a} D v^{i}$. Applying this on (1.10), we get

$$
D v_{l} j=P_{i}^{j}\left(-\kappa_{l} v_{l-1}^{i}+\kappa_{l+1} v_{l+1}^{i}\right)+v_{l}^{q} Q_{q}^{b} D \delta_{b}^{j}
$$

with respect to $l=0, \ldots, n-1 ; \kappa_{0}=0 ; \kappa_{n}=0$. Then we can now state the following theorem [3].

Theorem 1.2. Let us take a point $M$ of the curve $C$ in the $R-O_{n}$ space and $v_{0}, v_{1}, \ldots, v_{n-1}$ are the mutually orthogonal unit vectors that satisfy the relations (1.10) and (1.11) so that $\kappa_{0}=0$ and $\kappa_{n}=0$ hold. Hence we obtain (1.12) by applying the covariant differential $D$ on the covariant components of the observed vectors [3]. 
Remark 1.1.1. The relation (1.12) is the Frenet formula with respect to the covariant differential ${ }^{\prime} D$, applied on the contravariant components of the vectors.

If we apply the basic covariant differential " $\bar{D}$ to the tangent vectors $v_{0}^{i}$ and $v_{0}^{j}$, we obtain the known Frenet formulas for the Riemannian case so we omit this version of the study.

\section{Frenet Formula in $R-O_{3}$ and Slant Helices}

According to observation above, we may express the following theorem for dimension 3 by a close analogy with Theorem 1.1

Theorem 2.1. If $C: x^{3}(s)$ is the curve of an $R$ - $O_{3}$ space and $v_{l}, l=0,1,2$ are mutually orthogonal unit vectors which satisfies

$$
D v_{l} j=P_{i}^{j}\left(-\kappa_{l} v_{l-1}^{i}+\kappa_{l+1} v_{l+1}^{i}\right)+v_{l}^{q} Q_{q}^{b} D \delta_{b}^{j}
$$

and $v_{4}$ is the unit orthogonal to all before and $\kappa_{0}=0, \kappa_{3}=0$ holds then the vector $v_{3}$ satisfies equation (2.1), too.

Thus we obtain Frenet trihedron as follows

$$
\begin{aligned}
& D v_{0} j=P_{i}^{j}\left(\kappa_{1} v_{1}^{i}\right)+v_{0}^{q} Q_{q}^{b} D \delta_{b}^{j}, \\
& D v_{1} j=P_{i}^{j}\left(-\kappa_{1} v_{0}^{i}+\kappa_{2} v_{2}^{i}\right)+v_{1}^{q} Q_{q}^{b} D \delta_{b}^{j}, \\
& D v_{2} j=P_{i}^{j}\left(-\kappa_{2} v_{1}^{i}\right)+v_{2}^{q} Q_{q}^{b} D \delta_{b}^{j} .
\end{aligned}
$$

Using matrix expression we get

$$
\left[\begin{array}{l}
D v_{0} j \\
D v_{1} j \\
D v_{2} j
\end{array}\right]=P_{i}^{j}\left[\begin{array}{ccc}
0 & \kappa_{1} & 0 \\
-\kappa_{1} & 0 & \kappa_{2} \\
0 & -\kappa_{2} & 0
\end{array}\right] \cdot\left[\begin{array}{c}
v_{0}^{i} \\
v_{1}^{i} \\
v_{2}^{i}
\end{array}\right]+Q_{q}^{b} D \delta_{b}^{j}\left[\begin{array}{c}
v_{0}^{q} \\
v_{1}^{q} \\
v_{2}^{q}
\end{array}\right] .
$$

With these preparatory remarks we give the following

Definition 2.1. A unit speed curve $\alpha$ is called a slant helix if there exists a constant vector field $U$ in $\mathrm{R}-\mathrm{O}_{3}$ such that the function $g_{i j}\left(v_{1} j, U\right)$ is constant.

This definition is motivated by what happens in Euclidean space. Recently Izumiya and Takeuchi [9] have introduced the slant helix in Euclidean space by saying that normal lines make constant angle with a fixed direction and they characterize a slant helix if and only if the function

$$
\frac{\kappa^{2}}{\left(\kappa^{2}+\tau^{2}\right)^{3 / 2}}\left(\frac{\tau}{\kappa}\right)^{\prime}
$$

is constant.

In this work, we focus on this subject and obtain new characterization for 3-dimensional Riemann-Otsuki space $\mathrm{R}-\mathrm{O}_{3}$. 
Firstly, we assume that $\alpha$ is a slant helix. Let $U$ be the vector field such that the function $g_{i j}\left(v_{1} j, U\right)=c$ is constant. There exists smooth functions $a_{1}, a_{2}$ and $a_{3}$ such that

$$
U=a_{1}(s) v_{0} j+a_{2}(s) v_{1} j+a_{3}(s) v_{2} j
$$

As $U$ is constant by differentiation (2.5) together (2.3) gives

$$
\begin{aligned}
a_{1}^{\prime}-P_{i}^{j}\left(\kappa_{1} a_{2}+a_{1} Q_{q}^{b} D \delta_{b}^{j}\right) & =0, \\
-P_{i}^{j}\left(-\kappa_{1} a_{1}+\kappa_{2} a_{3}+a_{2} Q_{q}^{b} D \delta_{b}^{j}\right) & =0, \\
a_{3}^{\prime}-P_{i}^{j}\left(\kappa_{2} a_{2}+a_{3} Q_{q}^{b} D \delta_{b}^{j}\right) & =0 .
\end{aligned}
$$

Using (2.6) and (2.8) we get

$$
a_{3}=\frac{\kappa_{1}}{\kappa_{2}} a_{1}-\frac{a_{2}}{\kappa_{2}} Q_{q}^{b} D \delta_{b}^{j} .
$$

Moreover

$$
g_{i j}(U, U)=a_{1}^{2}+a_{2}^{2}+a_{3}^{2}=\text { constant. }
$$

Taking into account of (2.8), (2.9) and (2.10) together and after routine calculations we set

$$
a_{1}^{2}\left(1+\left(\frac{\kappa_{1}}{\kappa_{2}}\right)^{2}-2 \frac{\kappa_{1}}{\kappa_{2}^{2}} Y+\frac{Y_{2}}{\kappa_{2}^{2}}\right)=\varepsilon m^{2}, \quad m>0, \quad \varepsilon \in\{-1,0,1\}
$$

where $Y$ and $Y_{2}$ are constants.

Suppose that $\varepsilon=0$ this means that $U$ is a constant vector and result is obvious. If $\varepsilon= \pm 1$ we get

$$
a_{1}^{2}= \pm \frac{m^{2}}{\left(1+\left(\frac{\kappa_{1}}{\kappa_{2}}\right)^{2}-2 \frac{\kappa_{1}}{\kappa_{2}^{2}} Y+\frac{Y_{2}}{\kappa_{2}^{2}}\right)}
$$

and

$$
a_{1}= \pm \frac{m}{\sqrt{\left(1+\left(\frac{\kappa_{1}}{\kappa_{2}}\right)^{2}-2 \frac{\kappa_{1}}{\kappa_{2}^{2}} Y+\frac{Y_{2}}{\kappa_{2}^{2}}\right)}}, \quad \text { or }
$$

$$
a_{1}= \pm \frac{m}{\sqrt{\left(\frac{\kappa_{1}}{\kappa_{2}}\right)^{2}-2 \frac{\kappa_{1}}{\kappa_{2}^{2}} Y+\frac{Y_{2}}{\kappa_{2}^{2}}-1}} \text {. }
$$

Then (2.6) yields

$$
\frac{d}{d s}\left[ \pm \frac{m}{\sqrt{\left(1+\left(\frac{\kappa_{1}}{\kappa_{2}}\right)^{2}-2 \frac{\kappa_{1}}{\kappa_{2}^{2}} Y+\frac{Y_{2}}{\kappa_{2}^{2}}\right)}}\right]=P_{i}^{j}\left(\kappa_{1} a_{2}+a_{1} Q_{q}^{b} D \delta_{b}^{j}\right)
$$


or

$$
\frac{d}{d s}\left[ \pm \frac{m}{\sqrt{\left(\left(\frac{\kappa_{1}}{\kappa_{2}}\right)^{2}-2 \frac{\kappa_{1}}{\kappa_{2}^{2}} Y+\frac{Y_{2}}{\kappa_{2}^{2}}-1\right)}}\right]=-P_{i}^{j}\left(\kappa_{1} a_{2}+a_{1} Q_{q}^{b} D \delta_{b}^{j}\right)
$$

on $I$. This can be written as

$$
\frac{\left(\left(\frac{\kappa_{1}}{\kappa_{2}}\right)^{2}-2 \frac{\kappa_{1}}{\kappa_{2}^{2}} Y+\frac{Y_{2}}{\kappa_{2}^{2}}-1\right)^{\prime}}{\left(\left(\frac{\kappa_{1}}{\kappa_{2}}\right)^{2}-2 \frac{\kappa_{1}}{\kappa_{2}^{2}} Y+\frac{Y_{2}}{\kappa_{2}^{2}}-1\right)^{3 / 2}= \pm \frac{P_{i}^{j}\left(\kappa_{1} a_{2}+a_{1} Q_{q}^{b} D \delta_{b}^{j}\right)}{m}}
$$

and

$$
\frac{\left(1+\left(\frac{\kappa_{1}}{\kappa_{2}}\right)^{2}-2 \frac{\kappa_{1}}{\kappa_{2}^{2}} Y+\frac{Y_{2}}{\kappa_{2}^{2}}\right)^{\prime}}{\left(1+\left(\frac{\kappa_{1}}{\kappa_{2}}\right)^{2}-2 \frac{\kappa_{1}}{\kappa_{2}^{2}} Y+\frac{Y_{2}}{\kappa_{2}^{2}}\right)^{3 / 2}}= \pm \frac{P_{i}^{j}\left(\kappa_{1} a_{2}+a_{1} Q_{q}^{b} D \delta_{b}^{j}\right)}{m} .
$$

Conversely, assume that the conditions (2.14) and (2.15) are satisfied. To simplify the calculations, we assume that (2.14) is constant, namely $a_{1}$ and $\kappa_{1}$. We define

$$
\begin{gathered}
U=\frac{\kappa_{2}}{\sqrt{1+\left(\frac{\kappa_{1}}{\kappa_{2}}\right)^{2}-2 \frac{\kappa_{1}}{\kappa_{2}^{2}} Y+\frac{Y_{2}}{\kappa_{2}^{2}}}} v_{0} j+P_{i}^{j}\left(\kappa_{1} a_{2}+a_{1} Q_{q}^{b} D \delta_{b}^{j}\right) v_{1} j \\
+\left[\frac{\kappa_{1}}{\sqrt{1+\left(\frac{\kappa_{1}}{\kappa_{2}}\right)^{2}-2 \frac{\kappa_{1}}{\kappa_{2}^{2}} Y+\frac{Y_{2}}{\kappa_{2}^{2}}}}-a_{2} \frac{Q_{q}^{b} D \delta_{b}^{j}}{\kappa_{2}}\right] v_{2} j
\end{gathered}
$$

A differentiation of (2.16) together Frenet equations gives $\frac{d U}{d s}=0$, that is, $U$ is a constant vector. On the other hand taking into account $a_{1}$ and $\kappa_{1}$ are constant $g\left(v_{1} j, U\right)=$ cons $\tan t=c$ and this means that $\alpha$ is a slant helix. Hence we have proved the following theorem.

Theorem 2.2. Let $\alpha$ be unit speed curve in $R-O_{3}$. Then $\alpha$ is a slant helix if and only if either one the next two functions

$$
\frac{\left(\left(\frac{\kappa_{1}}{\kappa_{2}}\right)^{2}-2 \frac{\kappa_{1}}{\kappa_{2}^{2}} Y+\frac{Y_{2}}{\kappa_{2}^{2}}-1\right)^{\prime}}{\left(\left(\frac{\kappa_{1}}{\kappa_{2}}\right)^{2}-2 \frac{\kappa_{1}}{\kappa_{2}^{2}} Y+\frac{Y_{2}}{\kappa_{2}^{2}}-1\right)^{3 / 2}}
$$


or

$$
\frac{\left(1+\left(\frac{\kappa_{1}}{\kappa_{2}}\right)^{2}-2 \frac{\kappa_{1}}{\kappa_{2}^{2}} Y+\frac{Y_{2}}{\kappa_{2}^{2}}\right)^{\prime}}{\left(1+\left(\frac{\kappa_{1}}{\kappa_{2}}\right)^{2}-2 \frac{\kappa_{1}}{\kappa_{2}^{2}} Y+\frac{Y_{2}}{\kappa_{2}^{2}}\right)^{3 / 2}}
$$

is constant everywhere $\left(\frac{\kappa_{1}}{\kappa_{2}}\right)^{2}-2 \frac{\kappa_{1}}{\kappa_{2}^{2}} Y+\frac{Y_{2}}{\kappa_{2}^{2}}-1$ does not vanish.

Remark 2.1. The authors don't know what happens if

$$
\left(\frac{\kappa_{1}}{\kappa_{2}}\right)^{2}-2 \frac{\kappa_{1}}{\kappa_{2}^{2}} Y+\frac{Y_{2}}{\kappa_{2}^{2}}-1
$$

vanishes in some points. On the other hand if we take covariant part of the connection the Frenet formula of the $\mathrm{R}-\mathrm{O}_{3}$ space are not different from the known Frenet formulas of 3-dimensional Euclidean space. Hence we get the same results with [9] for this type.

\section{References}

[1] A.O. Ogrenmis, M. Ergut, M. Bektas, On the helices In the Galilean Space $G_{3}$, Iranian Journal of Science and Technology, Transaction A, Vol. 31, No. A2 (2007), 177-181.

[2] A.T. Ali, R. Lopez, On slant helices in Minkowski space $E_{1}^{3},(2008)$, http://arxiv.org/pdf/0810.1464.pdf

[3] F.D. Nadj, The Frenet Formulae of the Riemann-Otsuki Space, Review of Research Fac. of. Sci. University of Novi Sad, Mathematics, Vol. 16, No. 1 (1986), 95-106.

[4] F.D. Nadj, Autoparallel curves of Riemann-Otsuki spaces, Review of Research Fac. of. Sci. University of Novi Sad, Mathematics, Vol. 13, No. 1 (1983), 228-243.

[5] H. Balgetir, M. Bektas, M. Ergut, On a characterization of null helix, Bulletin of the Institute of Mathematics Academia Sinica, Vol. 29, No. 1 (2001), 71-78.

[6] H.B. Karadag, M. Karadag, Null Generalized slant helices 4-dimensional Lorentzian Spaces, Differential Geometry Dyn. Sys., 10 (2008), 178-185.

[7] M. Külahcı, M. Bektaş, M. Ergüt, On Harmonic curvatures of a Frenet curve in Lorentzian space, Chaos, Solitons and Fractals, 42 (2009), 1668-1675.

[8] M. Yildirim Yilmaz, M. Bektas, General Properties of Bertrand curves in RiemannOtsuki Space, Nonlinear Analysis, Theory methods and Appl., 69 (2008), 3225-3231.

[9] S. Izumiya, T. Nobuko, New special Curves and Developable surfaces, Turk. J. Math., 28 (2004), 153-164. 
MÜNEVVER Yildirim Yilmaz

FirAT UNIVERSITY

Faculty of Science

Department of Mathematics

23119 ELAZIĞ

TÜRKIYE

E-mail address: myildirim@firat.edu.tr

Mihriban KÜlahCI

Firat University

FACULTy OF Science

Department of Mathematics

23119 ELAZIĞ

TÜRKIYE

E-mail address: mihribankulahci@gmail.com

Alper O. ÖĞRenMiş

Firat University

Faculty of Science

Department of Mathematics

23119 ElazIĞ

TÜRKIYE

E-mail address: ogrenmisalper@gmail.com 